\title{
Synthesis and Characterization Benzimidazole Ring by Using O-phenylinediamine with Different Compounds and Using Mannich reaction for Preparation of Some Derivatives
}

\author{
ABDULLAH JAWAD KADHIM \\ Chemistry Department, College of Education, University of Al-Qadisiyah, Iraq 2016-2017 \\ Corresponding author E-mail: Abdullah.Kadhim@qu.edu.iq \\ http://dx.doi.org/10.13005/ojc/340152
}

(Received: July 24, 2017; Accepted: October 02, 2017)

\begin{abstract}
The research includes synthesis and characterization Benzimidazole rings by using different compounds such as Urea, Thiourea and Carboxylic acid by reactant with O-phenylinediamine, then substitution hydrogen atom with present on nitrogen atom by reactant with primary and secondary amines according to Mannich reaction. Compounds was organized by using F.T.I.R and HNMR spectroscopy.
\end{abstract}

Keywords: Benzimidazole, O-phenylinediamine, Thiouoria, Amines, Mannich reaction.

\section{INTRODUCTION}

Benzimidazole is heterocyclic compound found in many natural and non-natura1 products, such as some vitamins ${ }^{1}$. Therefore, benzimidazole substitutes tooks the attention of differents research groups, especially as compensation or replacement in the position 1,2 is very important sites in the impact of drug effective ${ }^{2}$. In This study reported some of the ways to prepare benzimidazole 2-substitution, for the importance of this compound in the field of antibiotics,such as cancer,angiotensin-II receptor antegonests and antimicrobial properties ${ }^{3}$ antifungal, antiparkinson, ...etc ${ }^{4}$. The $\mathrm{NH}$ group compounds are able to entering into $\mathrm{N}$-alkylation and $\mathrm{N}$-acylation according to Mannich and Michael reaction as in isatin compounds ${ }^{5}$, which are similar with benzimidazoles. Mannich reaction very important reaction by converting some of the prepared compounds into other compounds are more important than in the biological field ${ }^{6}$.

\section{MATERIALS AND METHODS}

1- $\quad$ Melting points were determined by using Melting PointSMP3 apparatus.

2- $\quad$ F.T.I.R. spectra were recorded by using Fourier Transform Infrared Spectrophotometer 
(F.T.I.R) $8400 \mathrm{~S}$ Shimadzu apparatus.

3- $\quad$ NMR Spectrometer $400 \mathrm{MHz}$, Avance III 400 Bruker, Germany.

\section{EXPERIMENTAL}

Synthesis 1,3-dihydro-benzimidazol-2-subsititution ( $A$ and $B$ )

A mixture of o-phenylenediamine with urea to yield (A) and with thiourea to yield (B) in existence of $\mathrm{HCl}$ in equal concentrations was heated at $130{ }^{\circ} \mathrm{C}$ under reflux in alcohol solution until the evolution of ammonia ceased ${ }^{7}$.

\section{Synthesis 2-Phenol-1H-benzimidazol (C)}

A mixture of equal concentration $(0.01 \mathrm{~mol})$ of o-phenylenediamine and Salicylic acid in $4 \mathrm{~N} \mathrm{HCl}$ $(20 \mathrm{ml})$ was refluxed for $30 \mathrm{~min}$. then cooled, filtered and recrystallized from absolute alcohol ${ }^{3}$.
General procedure for synthesis of compounds (A1) (B1) (C1)

A mixture of alcoholic solution with $(A)$, with (B), with (C) (0.01 mol) and formaldehyde (15 ml, $40 \%)$ was added slowly on alcoholic solution of (4-chloro-aniline ) $(0.01 \mathrm{~mol})$ the reaction mixture was stirred for three hours at room temperature and kept full night in the fridge. The solid obtained was filtered, washed with cold ethanol, dried and crystallized form aqueous ethanol to give compounds (A1), (B1), (C1).

\section{General procedure for the synthesis of compounds} (A2) (B2) (C2)

A mixture equimolar of $(A),(B),(C)$ with Diallylamine in presence formaldehyde were carried out $0-5{ }^{\circ} \mathrm{C}$ by stirring with magnetic stirrer 9 .

Table. 1: show physical properties of compounds

\begin{tabular}{lccccc}
\hline Compound & Molecular formula & Solvent & Yield \% & m.p. C & Color \\
\hline A & $\mathrm{C}_{7} \mathrm{H}_{6} \mathrm{~N}_{2} \mathrm{O}$ & Ethanol & 94 & $275-279$ & Yellow \\
B & $\mathrm{C}_{7} \mathrm{H}_{6} \mathrm{~N}_{2} \mathrm{~S}$ & Ethanol & 66 & $114-118$ & Violet \\
$\mathrm{C}$ & $\mathrm{C}_{13} \mathrm{H}_{10} \mathrm{~N}_{2} \mathrm{O}$ & Ethanol & 72 & $155-161$ & Violet \\
A1 & $\mathrm{C}_{21} \mathrm{H}_{18} \mathrm{~N}_{4} \mathrm{OCl}$ & Ethanol & 63 & Oil & Yellow \\
B1 & $\mathrm{C}_{14} \mathrm{H}_{12} \mathrm{~N}_{3} \mathrm{ClS}$ & Ethanol & 62 & Oil & Nutty \\
C1 & $\mathrm{C}_{20} \mathrm{H}_{16} \mathrm{~N}_{3} \mathrm{ClO}$ & Ethanol & 64 & Oil & Nutty \\
A2 & $\mathrm{C}_{21} \mathrm{H}_{28} \mathrm{~N}_{4} \mathrm{O}$ & Ethanol & 70 & Oil & Yellow \\
B2 & $\mathrm{C}_{14} \mathrm{H}_{17} \mathrm{~N}_{3} \mathrm{~S}$ & Ethanol & 73 & Oil & Nutty \\
C2 & $\mathrm{C}_{20} \mathrm{H}_{21} \mathrm{~N}_{3} \mathrm{O}$ & Ethanol & 76 & Oil & Nutty \\
\hline
\end{tabular}
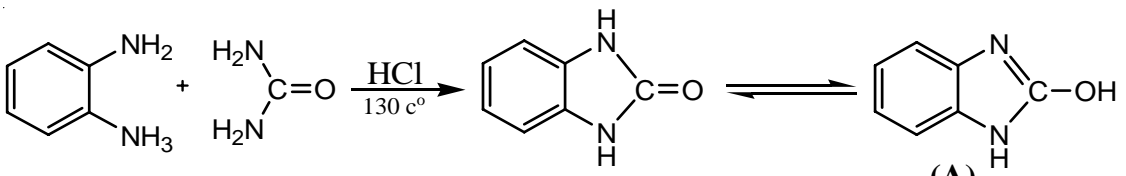<smiles></smiles>

(A2)<smiles>Cc1ccc(NCn2c(O)nc3ccccc32)cc1</smiles>

(A1)

\section{Scheme 1}


<smiles>Nc1ccccc1N</smiles><smiles>NC(N)=[SH][Mg]C(Cl)c1ccc2nc(S)[nH]c2c1</smiles>

(B)<smiles>CCCCN(C)Cn1c(S)nc2ccccc21</smiles>

(B2)<smiles>Nc1ccc(Cl)cc1CN1N=C(S)N(CNc2ccc(Cl)cc2)c2ccccc21</smiles>

(B1)

Scheme 2<smiles>Nc1ccccc1N</smiles><smiles>O=C(O)c1ccc(C=[W]Cl)cc1O</smiles><smiles>Oc1ccccc1-c1nc2ccccc2[nH]1</smiles>

(C)<smiles>CC=CCN1N=C(c2ccccc2O)N(CC#CCCC)c2ccccc21</smiles>

(C2)<smiles>Nc1ccc(Cl)cc1</smiles><smiles></smiles>

(C1)

\section{Scheme 3}

\section{RESULT AND DISCUSSION}

The aim of the research is to synthesis mannich bases from different Benzimidazole rings with primary and secondary amines by using formaldehyde as catalyst.
1H-benzimidazole-2-ol (A)

Infrared spectroscopy of this compound showed a broadband at $3348-3433 \mathrm{~cm}^{-1}$ refers to $\mathrm{O}-\mathrm{H}$ group, as well as the emergence of weak peak at $1739 \mathrm{~cm}^{-1}$ indicate that $\mathrm{O}-\mathrm{H}$ group turn to $\mathrm{C}=\mathrm{O}$ 
group by resonance between $\mathrm{O}-\mathrm{H}$ and $\mathrm{N}$ atom, the spectroscopy showed too other peaks at $3132-3178 \mathrm{~cm}^{-1}$ refers to $\mathrm{N}-\mathrm{H}$ group and at 3024 $\mathrm{cm}^{-1}$ to $\mathrm{C}-\mathrm{H}$ aromatic. Table (2) shows other peaks to this compound. ${ }^{1} \mathrm{HNMR}(400 \mathrm{MHz}, \mathrm{DMSO}) \delta(\mathrm{ppm})$ $\operatorname{Ar}(6.97 \mathrm{~Hz} 1 \mathrm{H}), \mathrm{OH}(5.45 \mathrm{~Hz} 1 \mathrm{H}), \mathrm{NH}(5.45 \mathrm{~Hz} 1 \mathrm{H})$

\section{1-\{[(4-chlorophenyl)amino]methyl\}-1H-benzimida zol-2-ol (A1)}

Infrared spectroscopy showed overlap of $(\mathrm{O}-\mathrm{H})$ peak with $(\mathrm{N}-\mathrm{H})$ peaks at $3261-3481 \mathrm{~cm}^{-1}$ after substitution $(\mathrm{H})$ atom by compound (4-chloro aniline), also emergence absorption peak at $2921-2979 \mathrm{~cm}^{-1}$ refers to (C-H) aliphatic. Table (2) shows the other peaks for this compound. ${ }^{1} \mathrm{HNMR}(400 \mathrm{MHz}, \mathrm{DMSO}) \delta(\mathrm{ppm}) \operatorname{Ar}(7.09,7.10 \mathrm{~Hz}$ and 7.25$), \mathrm{OH}(5.24 \mathrm{~Hz}), \mathrm{CH} 2(5.77-5.81 \mathrm{~Hz})$, $\mathrm{NH}(3.84-3.88 \mathrm{~Hz})$.

\section{1-[(diallyl-amino)methyl]-1H-benzimidazol-2-ol (A2)}

Infrared measurements of this compound showed disappearance $(\mathrm{N}-\mathrm{H})$ peak duo to substitution $\mathrm{H}$ atom by compound (diallyl amine) and emergence new peak at 2908-2958 $\mathrm{cm}^{-1}$ refers to $(\mathrm{C}-\mathrm{H})$ and $(\mathrm{CH}=\mathrm{CH})$ respectively in addition to (O-H) group in 3355-3394 $\mathrm{cm}^{-1}$. Table. (2) shows other peaks to this compound. ${ }^{1} \mathrm{HNMR}(400 \mathrm{MHz}$, DMSO) $\delta(\mathrm{ppm}) \mathrm{Ar}(7.18,7.19 \mathrm{~Hz}$ and $7.32 \mathrm{~Hz})$, $\mathrm{OH}(4.89 \mathrm{~Hz}), \mathrm{CH}_{2}(4.76 \mathrm{~Hz}), \mathrm{CH}=(5.87 \mathrm{~Hz}), \mathrm{CH} 2$ $(3.41 \mathrm{~Hz}),=\mathrm{CH} 2(5.30$ and $5.43 \mathrm{~Hz})$.

\section{H-Benzimidazole-2-thiol (B)}

Infrared spectrum for this compound showed absorption peaks sharp at $3379 \mathrm{~cm}^{-1}$ indicate to $(\mathrm{N}-\mathrm{H})$ group, also appearance absorption peak at $2360 \mathrm{~cm}^{-1}$ indicate to (S-H) group. Table. (2) shows other absorption peaks for this compound. ${ }^{1} \mathrm{HNMR}$ (400 MHz , DMSO) $\delta$ (ppm) $\mathrm{Ar}$ (broad 7.197.28Hz $2 \mathrm{H}$ ) and (broad 7.64-7.73 Hz 2H),

\section{1-\{[(4-chlorophenyl)amino]methyl\}-Benzimidazole-2- thiol (B1)}

Infrared spectrum showed stay of absorption peaks of $(\mathrm{N}-\mathrm{H})$ group at $3224 \mathrm{~cm}^{-1}$ after substitution of $(\mathrm{H})$ atom by compound (4-chloroaniline) and emergence absorption peak at $2923 \mathrm{~cm}^{-1}$ indicate to (C-H) aliphatic. Table. (2) shows other absorption peaks for this compound. 1-[(diprop-2-en-1-ylamino)methyl]-1 Hbenzimidazole2-thiol (B2)

Infrared spectrum showed disappearance absorption peak of $(\mathrm{N}-\mathrm{H})$ after substitution of $(\mathrm{H})$ atom by compound (Diallylamine) and appearance absorption peak at $2950 \mathrm{~cm}^{-1}$ indicated to $(\mathrm{C}-\mathrm{H})$ aliphatic. Table. (2) shows other absorption peaks for this compound. ${ }^{1} \mathrm{HNMR}$ (400 MHz, DMSO) $\delta(\mathrm{ppm}) \operatorname{Ar}(7.27,7.31$ and $7.72 \mathrm{~Hz}), \mathrm{SH}(2.54 \mathrm{~Hz} 1 \mathrm{H})$, $\mathrm{CH} 2(4.702 \mathrm{H}), \mathrm{CH} 2-\mathrm{CH}=(3.51-3.52 \mathrm{~Hz} 4 \mathrm{H}), \mathrm{CH}=$ $(5.07 \mathrm{~Hz} 2 \mathrm{H}),=\mathrm{CH} 2(4.85-4.95 \mathrm{~Hz} 4 \mathrm{H})$.

\section{2-(2-hydroxyphenyl)-1H-Benzimidazol (Compound)} Infrared spectrum showed broadband at $3402-3421 \mathrm{~cm}^{-1}$ indicated to $(\mathrm{OH})$ group and absorption peak at $3236 \mathrm{~cm}^{-1}$ indicated to $(\mathrm{NH})$ group. Table. (2) shows other absorption peaks for this compound. ${ }^{~} \mathrm{HNMR}(400 \mathrm{MHz}, \mathrm{DMSO}) \delta(\mathrm{ppm})$ $\operatorname{Ar}(7.23,7.24$ and $7.77 \mathrm{~Hz})$ and $(6.77,6.89$ and 7.05), $\mathrm{OH}(6.74 \mathrm{~Hz}), \mathrm{NH}(6.77 \mathrm{~Hz})$.

2-(2-hydroxyphenyl)-1-\{[(4-chlorophenyl) amino]methyl\}-1 $\mathrm{H}$-Benzimidazol (Compound 1)

Infrared spectrum showed appearance broadband at $3317-3417 \mathrm{~cm}^{-1}$ indicated to $(\mathrm{OH})$ group and absorption peak at $3186 \mathrm{~cm}^{-1}$ indicated to $(\mathrm{NH})$ group and new absorption peak at $2981 \mathrm{~cm}^{-1}$ indicated to $(\mathrm{C}-\mathrm{H})$ aliphatic. Table. (2) shows other absorption peaks for this compound. ${ }^{1} \mathrm{HNMR}$ $(400 \mathrm{MHz}$, DMSO) $\delta(\mathrm{ppm}) \operatorname{Ar}(7.03),(7.19-7.21)$, $(7.76,7.39)$ and $(6.76,6.86 \mathrm{~Hz}), \mathrm{OH}(4.97 \mathrm{~Hz} 1 \mathrm{H})$, $\mathrm{CH} 2(5.49 \mathrm{~Hz}), \mathrm{NH}(4.14-4.17 \mathrm{~Hz} 1 \mathrm{H})$.

\section{2-(2-hydroxyphenyl)-1-[(diprop-2-enamino)} methyl]-1 H-Benzimidazol (Compound 2)

Infrared spectrum showed survival of broadband for $(\mathrm{OH})$ group at $3398 \mathrm{~cm}^{-1}$ and disappearance absorption peak of $(\mathrm{N}-\mathrm{H})$ for Benzimidazole ring duo to substitution hydrogen atom by formaldehyde and secondary amine and new absorption peaks at $2800-2977 \mathrm{~cm}^{-1}$ indicated to $\mathrm{C}-\mathrm{H}$ aliphatic in propene. Table. (2) shows other absorption peaks for this compound. ${ }^{1} \mathrm{HNMR}$ $(400 \mathrm{MHz}, \mathrm{DMSO}) \delta(\mathrm{ppm}) \operatorname{Ar}(7.25-7.29 \mathrm{~Hz})$ and $(7.32,7.44,7.70,7.79 \mathrm{~Hz}), \mathrm{OH}(4.79 \mathrm{~Hz})$, $\mathrm{CH} 2(4.68 \mathrm{~Hz}),=\mathrm{CH}(5.49-5.55 \mathrm{~Hz}), \mathrm{CH} 2-\mathrm{CH}=(3.67-$ $369 \mathrm{~Hz})$, $=\mathrm{CH} 2(5.16-5.18 \mathrm{~Hz})$. 


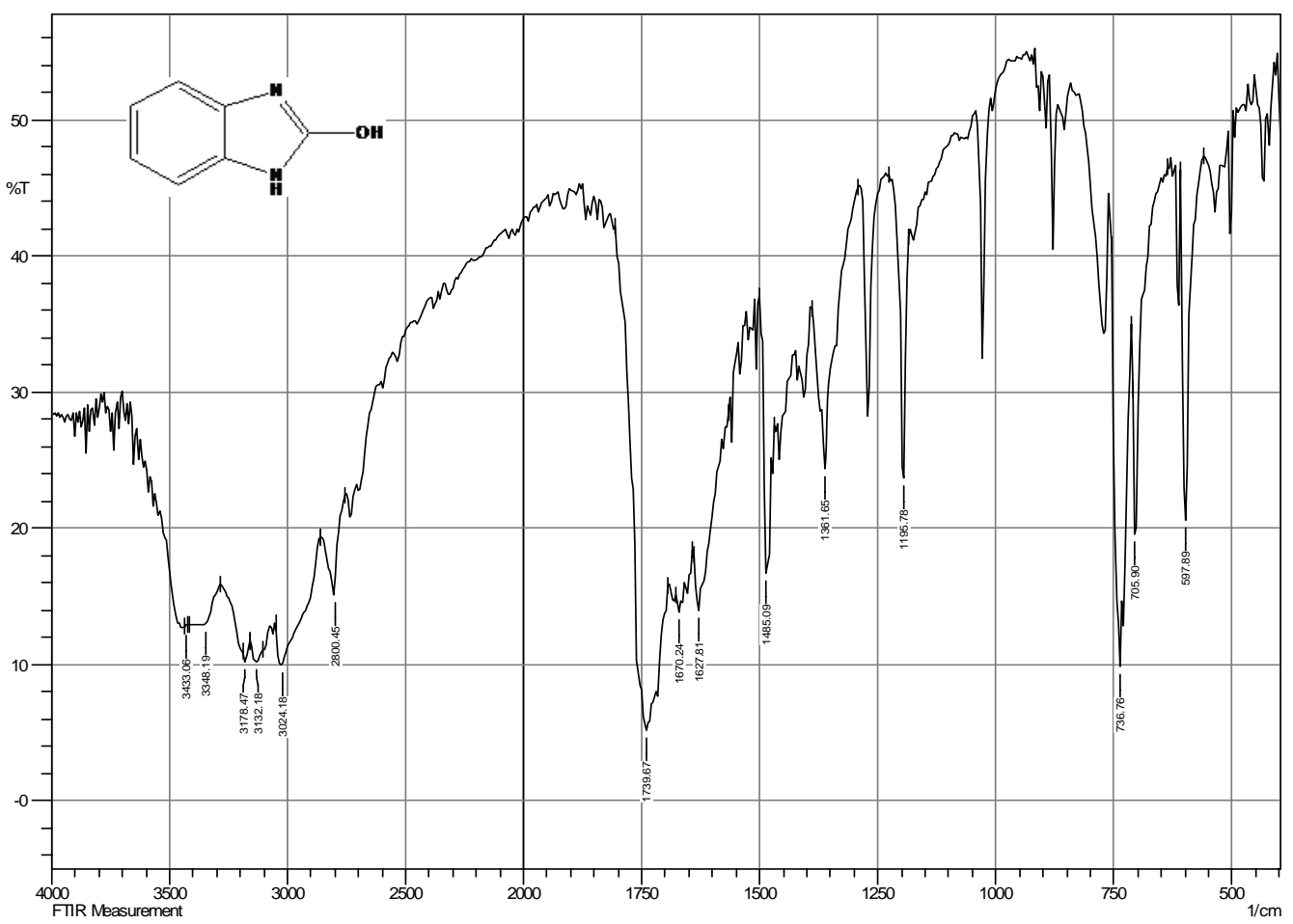

Fig. 1. F.T.I.R Spectroscopy of Compound (A)

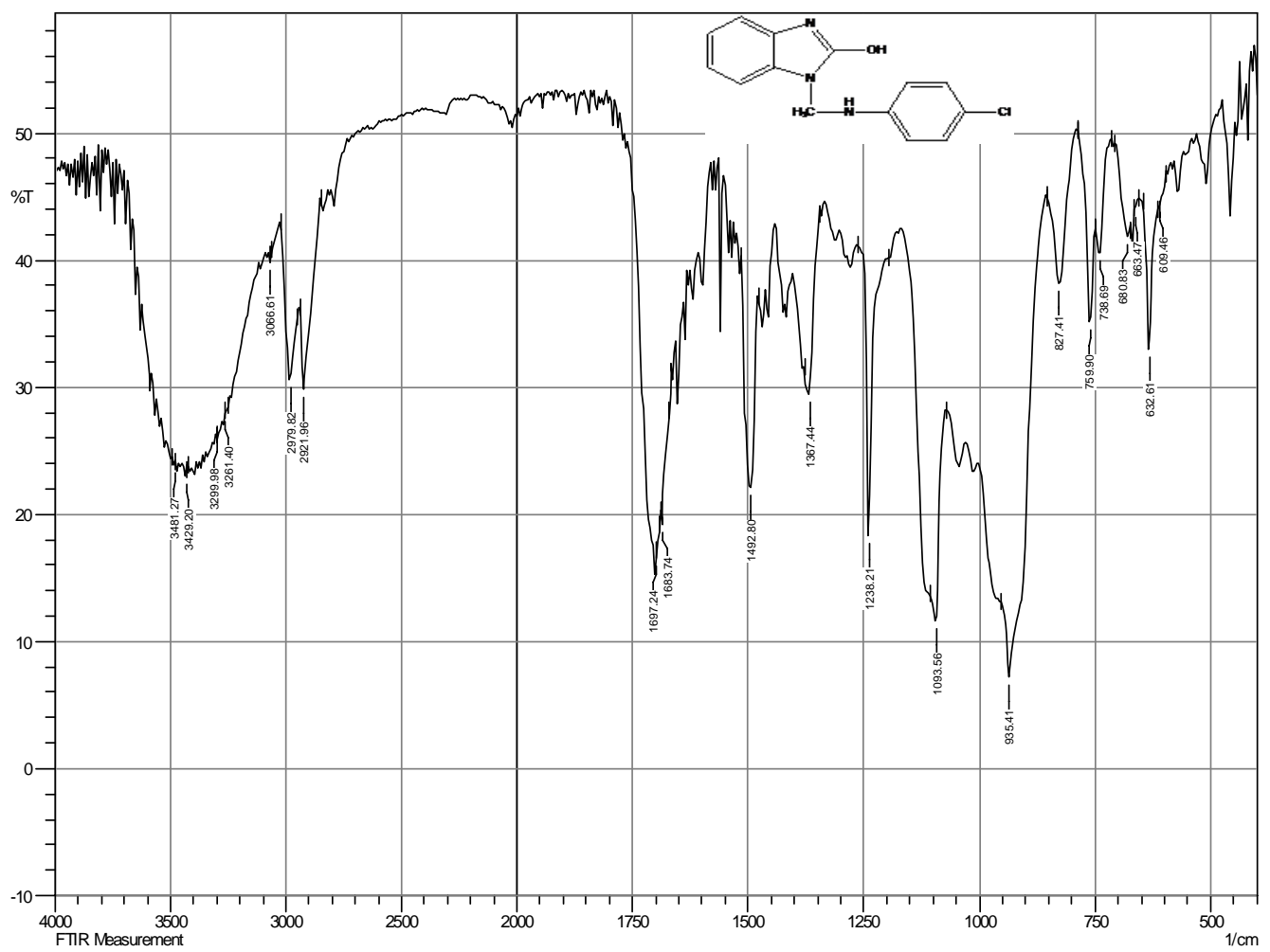

Fig. 2. F.T.I.R Spectroscopy of Compound (A1) 


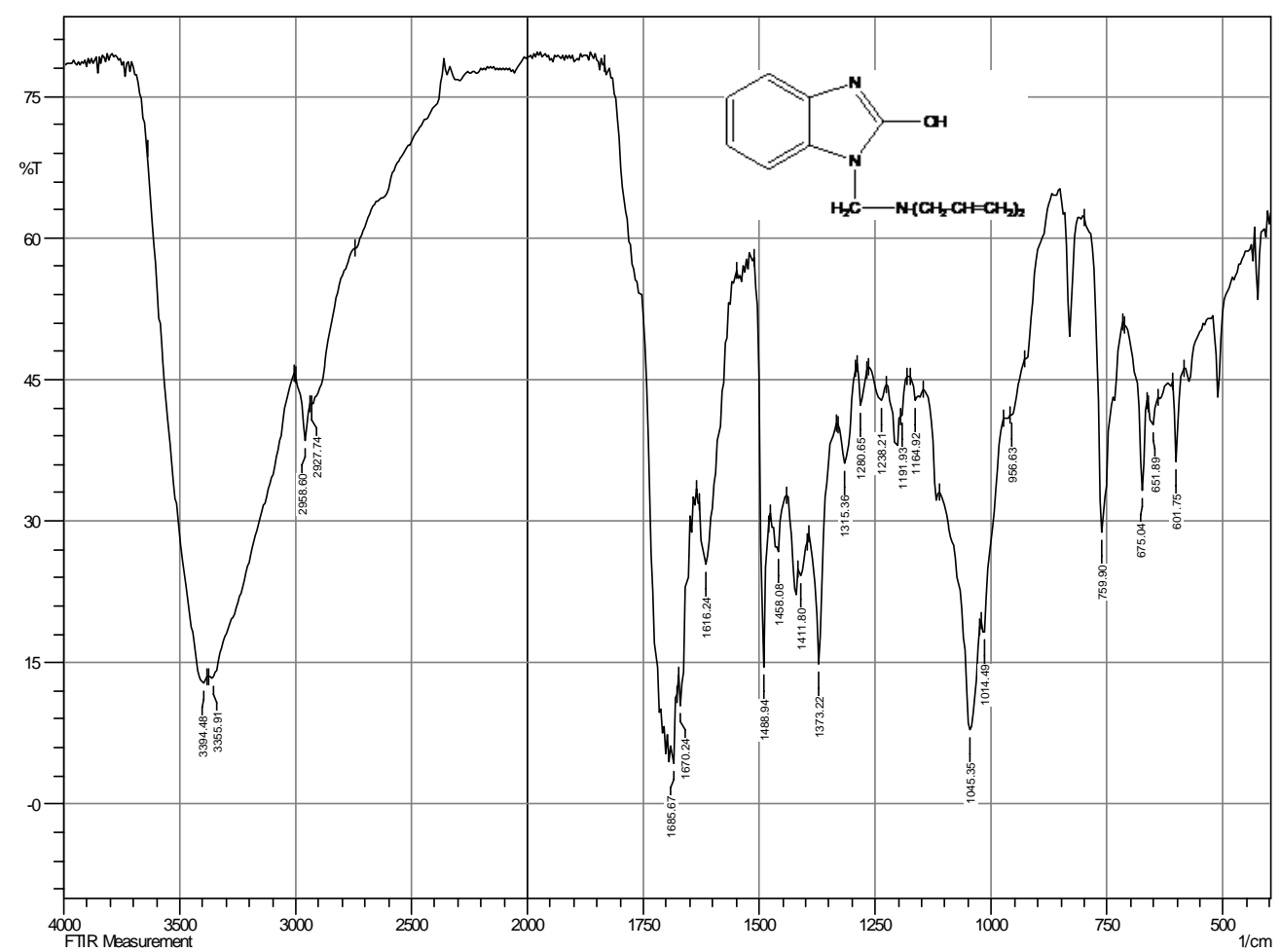

Fig. 3. F.T.I.R Spectroscopy of compound (A1)

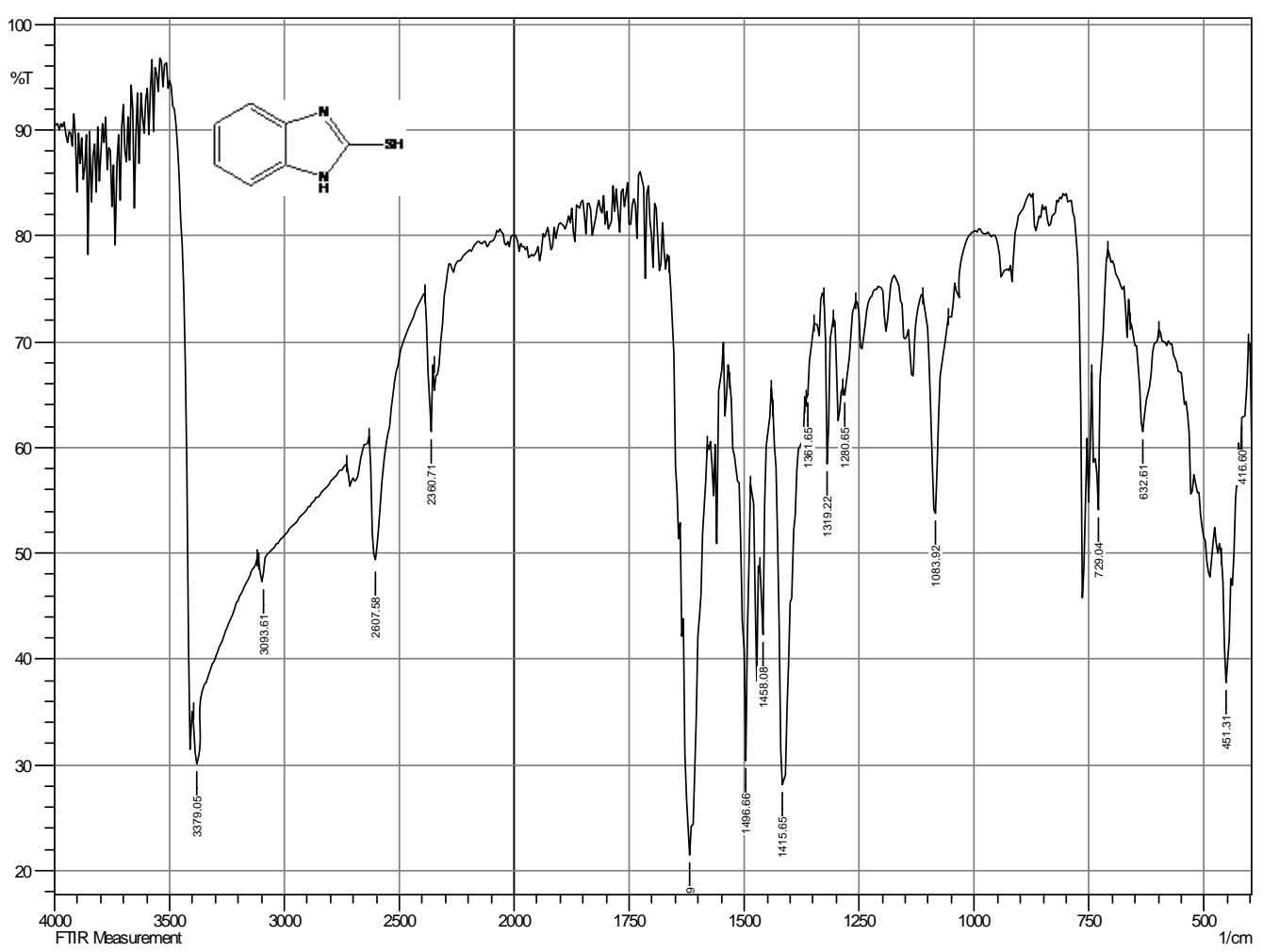

Fig. 4. F.T.I.R Spectroscopy of compound (B) 


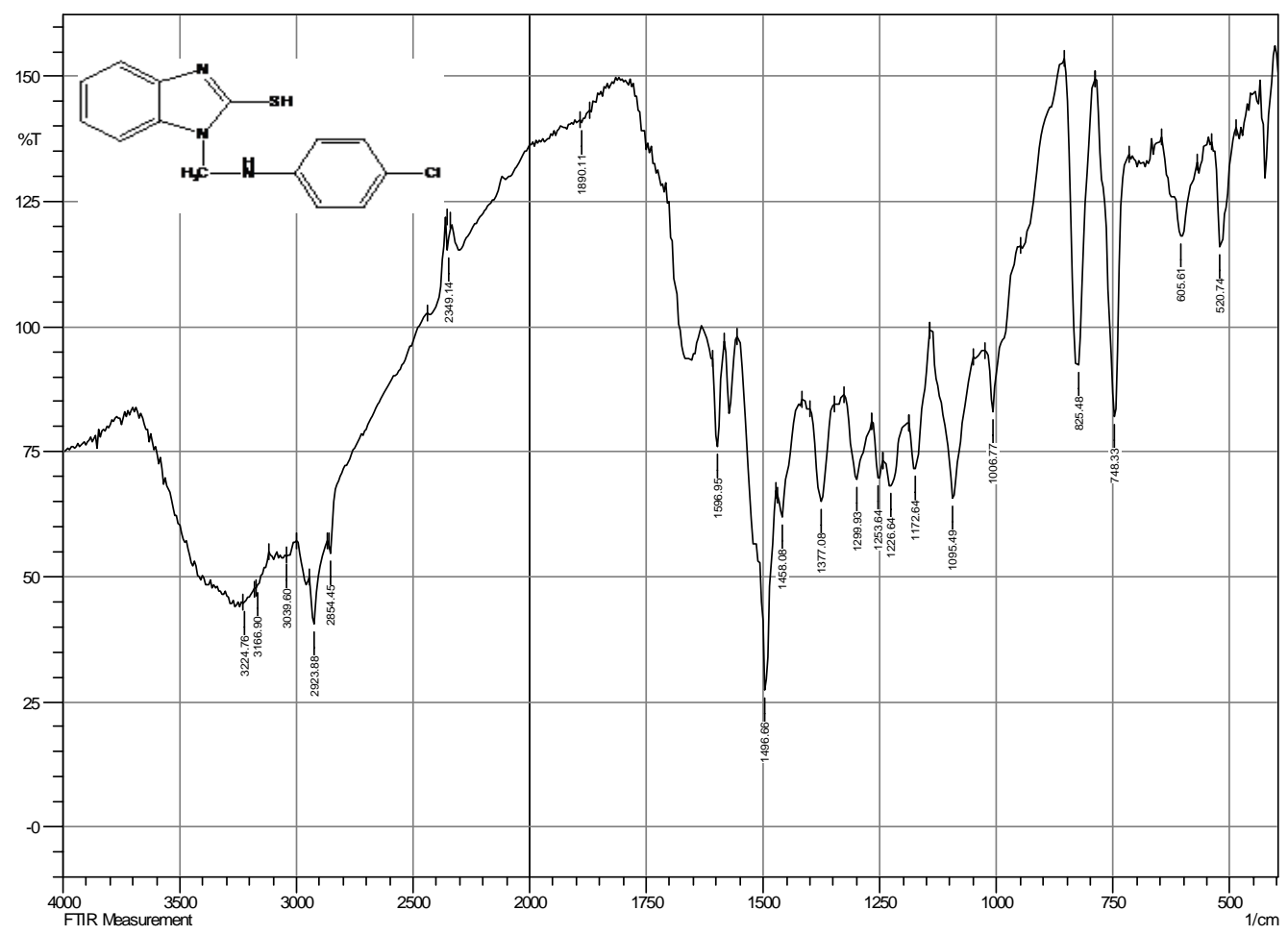

Fig. 5. F.T.I.R Spectroscopy of compound (B1)
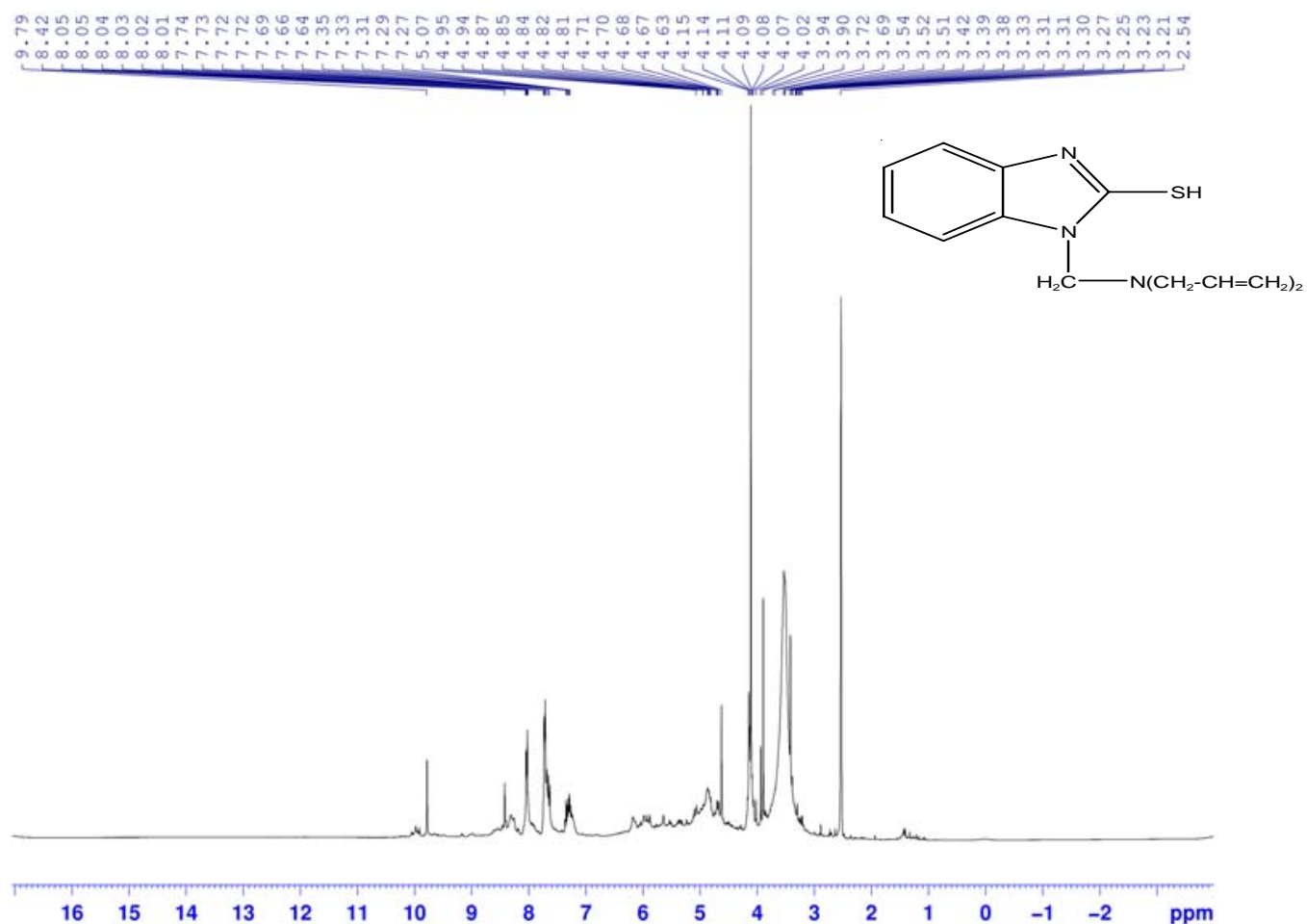

Fig. 6. H-NMR Spectroscopy of compound (B2) 


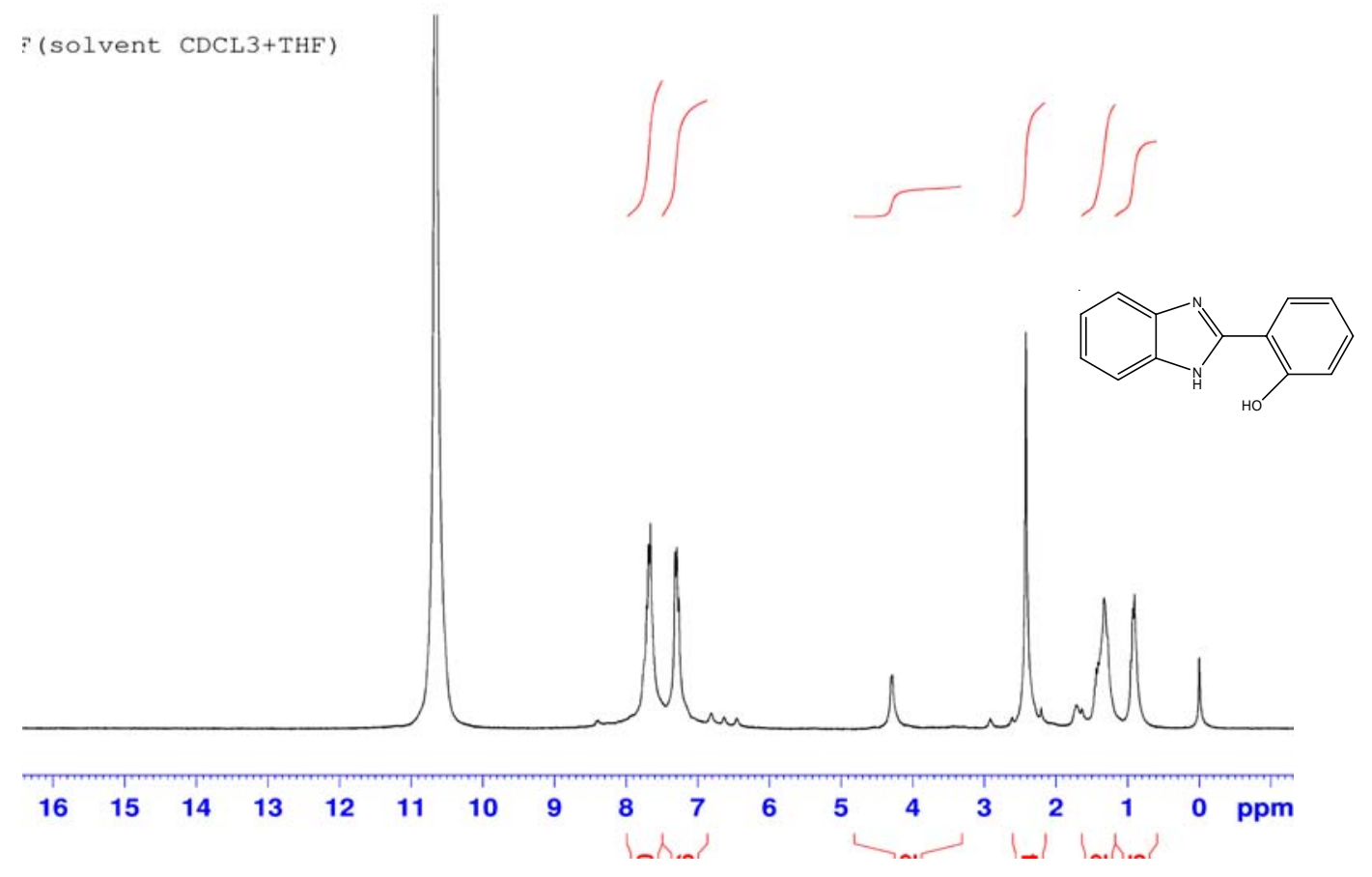

Fig. 7. H-NMR Spectroscopy of compound (C)

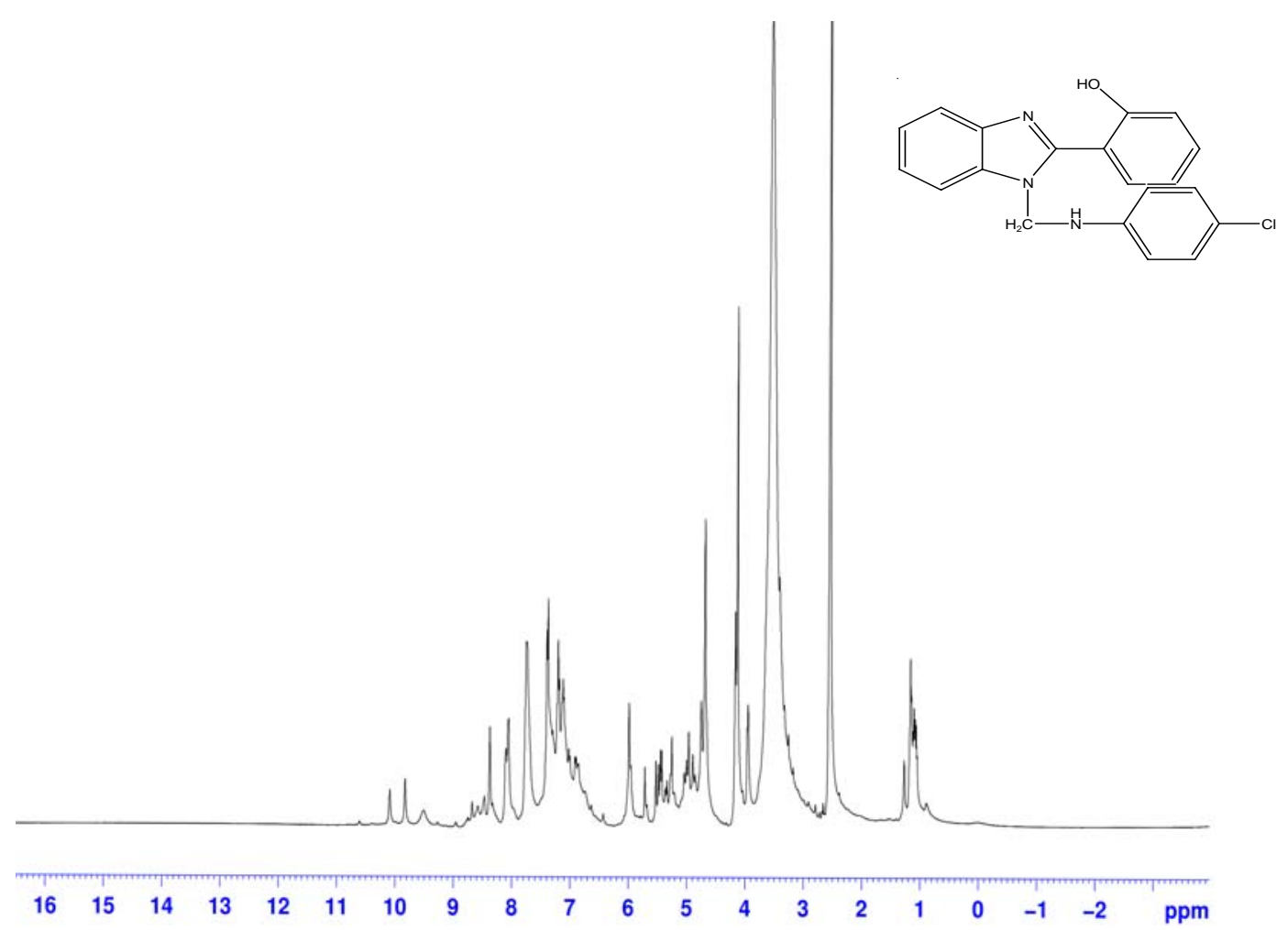

Fig. 8. H-NMR Spectroscopy of compound (C1) 


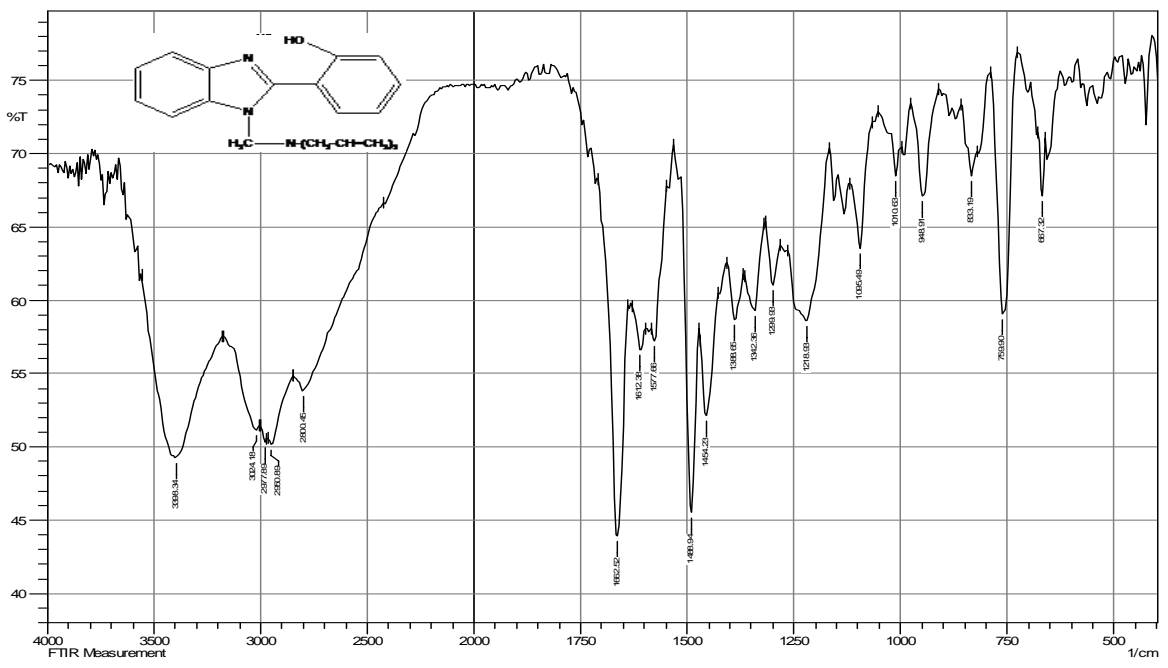

Fig. 9. F.T.I.R Spectroscopy of compound (C2)

Table. 2: Show F.T.I.R absorption packs of compounds

\begin{tabular}{|c|c|c|c|c|c|c|c|c|}
\hline Compounds & $\mathrm{C}-\mathrm{H}$ aryl & $\mathrm{C}=\mathrm{CC}=\mathrm{N}$ & $\mathrm{C}-\mathrm{N}$ & $\mathrm{C}-\mathrm{O}$ & C-S & $\mathrm{C}-\mathrm{Cl}$ & & \\
\hline A & 3024 & $1670-1627$ & 1361 & 1195 & - & - & - & $705-736$ \\
\hline B & 3093 & $1620-1496$ & 1280 & - & 1890 & - & - & 729 \\
\hline C & 3055 & 1654-1612 & 1249 & 1157 & - & - & - & 759 \\
\hline A1 & 3066 & 1697-1683 & 1238 & 1093 & - & 632 & 827 & 759 \\
\hline B1 & 3039 & $1630-1596$ & 1377 & - & 1890 & 605 & 825 & 748 \\
\hline C1 & 3066 & $1666-1573$ & 1584 & 1091 & - & 632 & 848 & 763 \\
\hline A2 & 3058 & $1685-1670$ & 1375 & 1045 & - & - & - & 759 \\
\hline B2 & 3055 & $1616-1542$ & 1272 & - & 1747 & - & - & 756 \\
\hline $\mathrm{C} 2$ & 3024 & $1662-1577$ & 1218 & 1095 & - & - & - & 759 \\
\hline
\end{tabular}

\section{CONCLUSION}

In this study I am reported synthesis of many Benzimidazol rings from o-phenylinediamineas starting material with different compounds by using $\mathrm{HCl}$ as catalyst in all synthesis works and note the higher of percentage ratio for the results, then using Mannich reaction to prepared derivatives of the Benzimidazol rings were prepared. These derivatives confirmed from spectral data analysis; F.T.I.R and H-NMR.

\section{ACKNOWLEDGEMENT}

The author gratefully acknowledges Chemistry department, University of Al-Qadissiah, Iraq for providing lab facilities to carry out this work.

\section{REFERENCE}

1. Ramanatham V., Sanjay D. V., Bobba. V. S. Kumar, Umesh N. B., Shekar B. B., and Uday C.Mashelkar. ARKIVOC., 2008 10(4), 27-49.

2. Shaimaa Adnan, Kasim Hassan and Hassan Thamer, Iraqi National Journal of Chemistry., 2014,53, 66-75.

3. Panneer Selvam, T., P. P. Radhika, S. Janagaraj1, A. Siva Kumar, Research in Biotechnology.,ISSN: 2229-791X, 2011, 2(3):50-57.

4. Mohan Babu Maradolla, Sunil Kumar Allam, Amaravathi Mandha, and G. V. P.Chandramouli,
ARKIVOC., 2008 10(5) 42-46.

5. Khalaf Ahmed Jasim AL- Bayati, Tikrit Journal of Pure Science., 2012, 17(2), ISSN: 1813 - 1662.

6. Suad M.Al-Araji and Rana A.Ali, Baghdad Science Journal., 2012, 9(1).

7. John B. Wright THF: Rsesarch Laboratories, The Upjohn Company, Kalamazoo, Michigan., 1951, 446 - 454.

8. Badie A. Ahmed and Salem J. Mohammed. Iraqi National Journal of Chemistry., 2011, 44, 582- 589.

9. V. Ravichandran, S. Mohan, and K. Suresh Kumar. ARKIVOC., 2007, 10(4), 51-57. 